\title{
The strategic development and strengthening of the profession of biokinetics
}

\author{
B K Grobler, ${ }^{1}$ BSc Biokinetics; T J Ellapen, ${ }^{2} \mathrm{PhD}$; Y Paul, ${ }^{2} \mathrm{PhD}$; G L Strydom, ${ }^{1} \mathrm{PhD}$ (emeritus professor) \\ ${ }^{1}$ School of Biokinetics, Recreation and Sport Science, Faculty of Health Sciences, North-West University, Potchefstroom, South Africa \\ ${ }^{2}$ Department of Sport, Rehabilitation and Dental Sciences, Faculty of Science, Tshwane University of Technology, Pretoria, South Africa
}

Corresponding author: B K Grobler(groblerkatryn@gmail.com)

Continuous planning for the future of any profession is wise. The profession of biokinetics has made remarkable advances since the 1980s. However, new opportunities for development need to be embarked on. This short commentary explores three such areas: training and education; entry into the South African public healthcare system; and interprofessional healthcare collaboration. The intention is to encourage the Biokinetics Association of South Africa and biokineticists to work together to attain these goals, thereby strengthening the profession.

Afr J Health Professions Educ 2021;13(1):8-9. https://doi.org/10.7196/AJHPE.2021.v13i1.1287

On 3 November 1982, the former South African (SA) Medical and Dental Council communicated to the committee of the heads of physical education departments at SA universities that biokinetics was officially acknowledged and included as a healthcare profession in the register of medical sciences. ${ }^{[1]}$ Subsequently, on 17 October 1987, the SA Association of Biokinetics was constituted, which was later renamed the Biokinetics Association of SA (BASA). ${ }^{[1]}$ The profession of biokinetics operates within the pathogenic and fortogenic healthcare paradigms, with a scope of profession (SoP) that involves improving the health and physical status of individuals through the prescription of structured exercise as derived from clinical assessments. ${ }^{[2,3]}$ The cardinal areas of practice involve final-phase rehabilitation and prevention of non-communicable diseases (NCDs), neuromusculoskeletal injuries, physical work capacity assessments and campaigning for health and wellness. ${ }^{[2,3]}$ Biokineticists are affiliated with their professional body (BASA) and their statutory body (the Health Professions Council of SA (HPCSA)). ${ }^{[4]}$ BASA supports the interests of students in training, intern biokineticists and practitioners, while the HPCSA protects the wellbeing of the public and guides the profession accordingly. ${ }^{[1,2]}$ Biokineticists operate within the SA private healthcare sector. The three primary areas of strategic development are: $(i)$ training and education; (ii) entry into the SA public healthcare system; and (iii) interprofessional healthcare collaboration.

\section{Training and education}

Currently, there are 12 biokinetics training institutions, of which six offer the traditional educational model (a 3-year undergraduate degree in human movement science (HMS) or equivalent, and a subsequent postgraduate specialisation in biokinetics). ${ }^{[5]}$ The other six institutions offer the new model, i.e. a 4-year professional degree. Although both models have similar academic content, it is imperative that all 12 institutions offer the 4-year professional degree. This degree is structured solely for the edification of biokinetics, while the traditional HMS undergraduate degree allows for specialisation in other subjects through subsequent postgraduate programmes. The professional degree has the advantage that students start their biokinetics internship (work-integrated learning) during their first year of study, affording them more time to hone their clinical expertise. Moreover, uniform compliance with the 4-year professional degree structure, similar to the academic requirements of physiotherapy and occupational therapy, reinforces the identity of this young profession. ${ }^{[4,5]}$ Training institutions should furthermore continue with evidence-based research, supporting the ethos of the profession (salutogenic effect of exercise). The evidence-based research must be published in a specialised biokinetics journal, similar to other therapeutic journals for the professions of physiotherapy and occupational therapy. ${ }^{[4]}$ The proposed journal must be accredited with the SA Department of Higher Education and Training and the Institute for Scientific Information, providing the opportunity for all subscribers to receive continuously updated information relevant to their professional development. The layout of the journal should be analogous to other professional journals, and therefore include original research and review articles, short communications, case studies, letters to the editor, announcements regarding upcoming conferences, as well as employment opportunities. ${ }^{[4]}$

\section{Entry into the South African public healthcare system}

The high prevalence of NCDs in SA places a great deal of pressure on the public healthcare system, which needs the support of and would benefit from the expertise of the biokinetics profession. ${ }^{[6]}$ Compliance with structured habitual physical activities to help support the healthcare management of coronary heart disease, hypertension, obesity, diabetes mellitus and chronic respiratory diseases is financially cheaper and effective ${ }^{[6,7]}$ Furthermore, the inclusion of biokineticists in the public healthcare sector will provide additional skilled clinicians who are able to collaborate with overworked members of the current healthcare system, thereby lessening the overall workload. One of SA's national healthcare strategies is to increase the physical activity of its citizens; this can be accomplished by the appointment of biokineticists at public hospitals, clinics and schools. 


\section{Interprofessional healthcare collaboration}

A third area of development is the encouragement of interprofessional healthcare collaboration. Respect for the SoP of fellow health professionals is firmly established through interprofessional education, ${ }^{[8]}$ which is a strong component of the training curriculum of international medical schools. This structure fosters an understanding and appreciation of the place of each profession within the healthcare paradigm, and aids in minimising the encroachment of the clinical SoP. ${ }^{[8]}$ Biokineticists are not primary healthcare practitioners (as opposed to general practitioners, medical specialists, nurses and physiotherapists); rather, they are finalphase therapists. ${ }^{[2,3]}$ Biokinetics training institutions, BASA and the HCPSA need to provide continuous education in this area. Furthermore, interprofessional collaboration should be extended with international affiliation to the World Confederation for Physical Therapy, as well as to various clinical exercise physiology, sports and rehabilitation medicine, national athletic training and kinesiotherapy associations, all of which are exercise-therapy professions. ${ }^{[8]}$ BASA should pursue international collaboration with exercise therapy professions in the USA, Canada, the UK, Asia, Australia, New Zealand, Namibia and Botswana.

\section{Conclusion}

A uniform 4-year professional biokinetics bachelor's degree will ensure a nationally aligned academic programme, mirroring other healthcare professions. Admission into the public healthcare system will provide much-needed professional exercise therapy-based management of NCDs and orthopaedic injuries, and strengthen the campaign for enhancing health and wellbeing through habitual exercise. Interprofessional collaboration, starting at tertiary institutions, will open the door for interprofessional respect and professionalism and eventually culminate in collaboration. Collectively, these strategies will strengthen the profession and improve the health and wellbeing of many South Africans.

\section{Declaration. None.}

Acknowledgements. None.

Author contributions. BKG and TJE conceptualised the idea of the article. BKG, TJE, YP and GLS drafted the article. BKG served as corresponding author. BKG and TJE amended the article as per reviewers' comments.

Funding. None.

Conflicts of interest. None.

\footnotetext{
1. Strydom GL. Biokinetics - the development of a health profession from physical education - a historical perspective. S Afr J Res Sport Phys Educ Recreat 2005;27(2):113-128.

2. Hall J. Scope of professions of physiotherapy, podiatry and biokinetics: Overlap identification. Physiother Podiatr Biokinet News 2013:8.

3. Strydom GL, Wilders CJ, Moss SI, Bruwer E. A conceptual framework of biokinetic procedures and referral system: An integrated protocol for the various health paradigms. Afr J Phys Health Educ Recreat Dance 2009;15(4):641-649.

4. Ellapen TJ, Swanepoel M. The evolution of the profession of biokinetics. S Afr J Res Sport Phys Educ Recreat 2017;39(1):41-49.

5. BASA - Biokinetics Association of South Africa: Guidelines for biokineticists. 2020. http://www.biokinetics.org za (accessed 30 January 2020)

6. Evans RW, Smith T, Kay P, et al. The need for biokineticists in the South African public health care system S Afr J Sports Med 2016;28(3):85-86. https://doi.org/10.17159/2078-516X/2016/v28i3a1310

7. Ehrman JK, Gordon PM, Visich PS, Keteyian SJ. Clinical Exercise Physiology. 3rd ed. Champaign, IL: Human Kinetics, 2013.

8. Ellapen TJ, Strydom GL, Swanepoel M, Hammill HV, Paul Y. Biokinetics. A South African Health Profession
} Evolving from Phyical Education and Sport. London: InTech, 2018.

Accepted 13 March 2020. 